\title{
Comparison and Analysis of PIM-DM and BST Multicasting Network over Drop Tail Queuing Technology
}

\author{
Ashish Kumar \\ NIT Jalandhar
}

\author{
Ajay K Sharma \\ NIT Jalandhar
}

\author{
Arun Singh \\ NIT Jalandhar
}

\begin{abstract}
The paper contrast a Multicasting network using PIM-DM (Protocol Independent Multicast - Dense Mode) with BST (BiDirectional Shared Tree) protocol using NS2. The networking topology is well analyzed for two sources and drop of data packet and throughput is recorded and drawn. The simulation results mark the decrease in drop out packets for BST by $55.88235 \%$ on node 0 and some increase in drop out packets at node 1 .
\end{abstract}

\section{General Terms}

Computer Network, Multicasting.

\section{Keywords}

PIM-DM, BST, Drop Tail, Data Multicasting, NS2, Tracegraph

\section{INTRODUCTION}

In 1990, Deering proposed IP multicast - an extension to the IP unicast service model for efficient multipoint communication [1]. It is a stateful service in that it requires routers to maintain State for forwarding multicast data toward receivers. It is a widely used service in today's computer networking system; it is mostly used in Streaming media, Internet television, video conferencing and net meeting etc. Routers involved in multicasting packets need a better management over stacking system of packets to be broadcasted. Quality of service (QOS) is dependent on the queuing algorithm used in the multicasting system. A PIM Domain is a contiguous set of routers that all implement PIM and are configured to operate within a common boundary defined by PIM Multicast Border Routers (PMBRs) [2]. It uses the idea that an actual delivery path to a node is the reverse of the path from the receiving node. Bi-directional delivery tree are built with CFR information towards the core [3]. In Shared Tree multicast routing, the tree is rooted at Rendezvous Point (RP). All the traffic is forwarded towards source and root (RP). The queuing algorithm used is Drop Tail. Drop Tail object, which implements First in First out (FIFO) scheduling and drop-on-overflow buffer management typical of most present day Internet routers [4].

\section{SYSTEM DESCRIPTION}

\subsection{TOPOLOGY}

A network of six nodes is created and UDP protocol is used to send constant bit rate (cbr) packets. The connection set up is as follows: node 1 is connected to node 2 ; node 2 is connected to node 3 ; node 2 is connected to node 4 ; node 2 is connected to node 4; node 3 is connected to node 4 ; node 4 is connected to node 5 ; node 4 is connected to node 6 ; node 5 is connected to node 6 ; bandwidth of link between node 2 and node 3 is $0.5 \mathrm{Mbps}$; bandwidth of link between node 4 and node 5 is $0.5 \mathrm{Mbps}$; bandwidth of link between node 4 and node 5 is
$0.5 \mathrm{Mbps}$; bandwidth of link between node 5 and node 6 is $0.5 \mathrm{Mbps}$, and all other connections have a bandwidth of $0.3 \mathrm{Mbps}$, delay of $10 \mathrm{~ms}$; node 1 and node 2 is the data source and multicast protocol will be put into effect at $0.4 \mathrm{~s}$ and $2 \mathrm{~s}$ respectively in the two node; receiver nodes $3,4,5$ and 6 will be effective at $0.6 \mathrm{~s}, 1.3 \mathrm{~s}, 1.6 \mathrm{~s}$, and $2.3 \mathrm{~s}$ respectively; node 4 and node 3 will leave the group at $1.9 \mathrm{~s}$ and $3.5 \mathrm{~s}$.

The node 1 and node 2 is the source node which refers to node 0 and node 1 in the topology and can be seen from the topology is as fig 1.Other nodes are marked as receivers. Node 2 is the Rendezvous point (RP) in BST multicasting

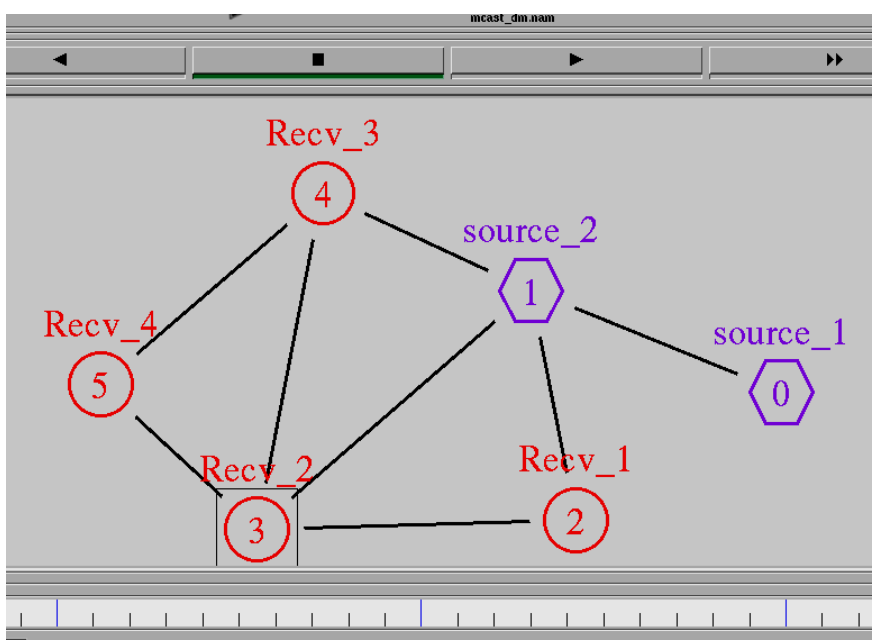

Fig. 1 Network Topology Design

\subsection{PIM-DM}

PIM-DM is a multicast routing protocol. PIM can use any routing protocol (RIP and OSPF, for instance) to maintain unicast routing, but it cannot transmit unicast by itself [5]. It uses unicast routing information base to flood multicast datagrams to all multicast routers connected in the network. It uses prune messages to prevent future messages from propagating to routers without group membership information. Dense mode (DM) refers to an environment where group members are relatively densely packed and bandwidth is plentiful [5].

It assumes that when a source starts sending, members in the network want to receive multicast datagrams. At the beginning multicast datagrams are flooded to whole network. PIM-DM uses RPF (Reverse path forwarding) to prevent looping of multicast datagrams while flooding and if some areas of the 
network do not have group members, PIM-DM will prune off the forwarding branch by instantiating prune state [6].

The prune message has a life time set with it. Once the lifetime expires, multicast datagram will be forwarded again to the previously removed/pruned branches.

Graft messages are used when a new member for a group appears in a pruned area. The router sends a graft message toward the source for the group to turn the pruned branch back into a forwarding branch for broadcast messages.

Commands used in configuring PIM-DM:

set group [Node allocaddr]

set mproto DM

set mrthandle [\$ns mrtproto \$mproto]

\subsection{BST}

BST is a multicasting protocol implemented in NS2 is in Research mode. BST uses tree structure to multicast traffic. In BST, multicast data can travel in both the direction of tree to reach receivers. When receivers are distributed throughout the network is gives the better result than other.

Bidirectional trees offer improved routing optimality by being able to forward data in both directions while retaining a minimum amount of state information [7]. RP used in this system is used to maintain the routing table for the upstream and downstream receivers. All the data is sent to the RP and RP then forwards is to the receivers using minimal path.

Commands used in configuring BST:

Set group [Node allocaddr]

BST set RP_(\$group) \$n(2)

\$ns mrtproto BST

In BST simulation we have set node 2 as RP to maintain state table.

\subsection{Drop Tail}

Drop Tail queuing method is by far the simplest approach to router queue management [8]. Routers decide when to drop packets. It uses first in first out algorithm. In Drop Tail, the traffic is not differentiated. Each packet is has the same priority. When the queue buffer is filled to its maximum capacity, the packets arrived afterward are dropped till the queue is full. That is, Drop Tail will keep discarding/dropping the packet until the queue has enough room for new packets.

The ns-2 implements enque and deque functions to implements queue and dequeue of packets. FIFO scheduling is implemented in the deque function by returning the first packet in the packet queue [9].

\section{PERFORMANCE EVALUATION}

\subsection{Simulating PIM-DM Multicasting} Network represented in 2D

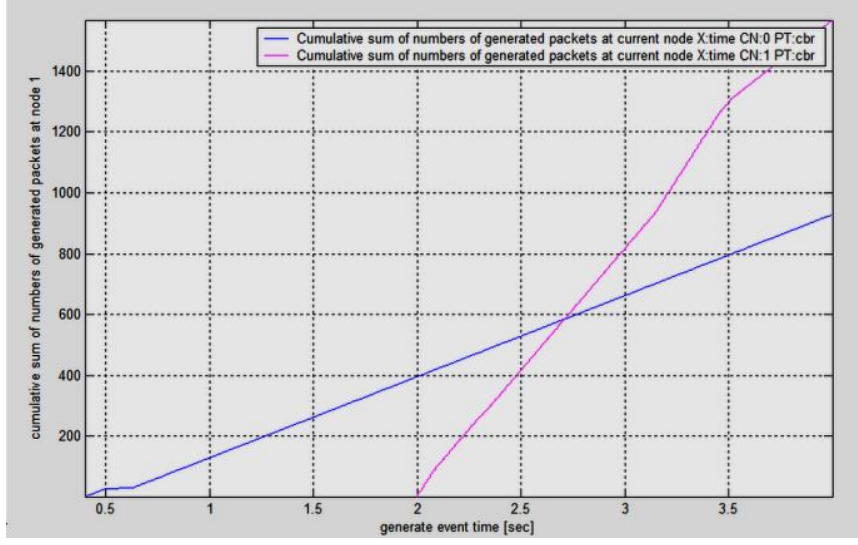

Fig.2 Packets Generated at Source $1 \& 2$

Figure 2 shows the total CBR packets generated at source 1 and source2. Source1 is represented by blue and source 2 by red. Source 2 starts generating packets after 2 seconds of source 1 and generated up to 1500 data packets, while source 1 up to 950 data packets (approx.)

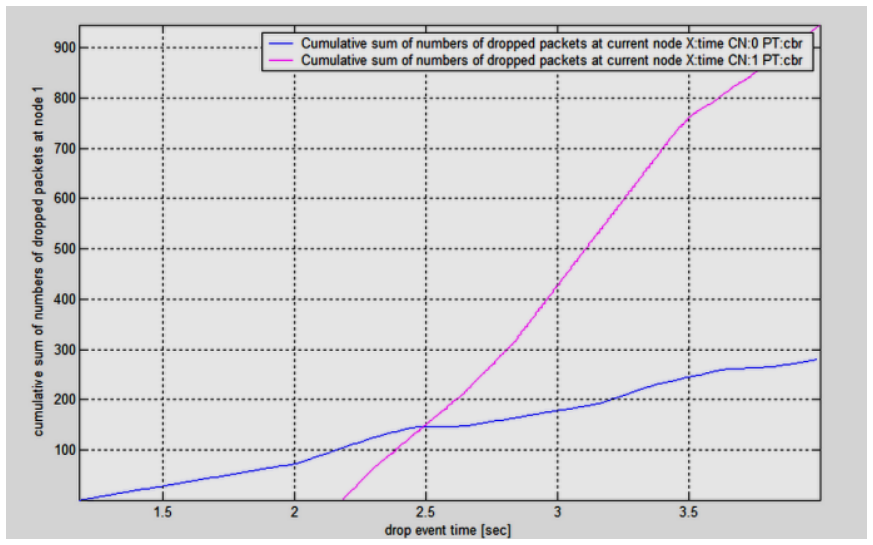

Fig.3 Packets dropped at Source $1 \& 2$

Figure 3 shows the drop out packets at source1 and source2 which is in the range of 280 and 945 respectively. Dropping of packets starts at source 2 after $2.2 \mathrm{~s}$ of start of the simulation as source 2 starts generating the packets and the traffic load increase at node 2 . 


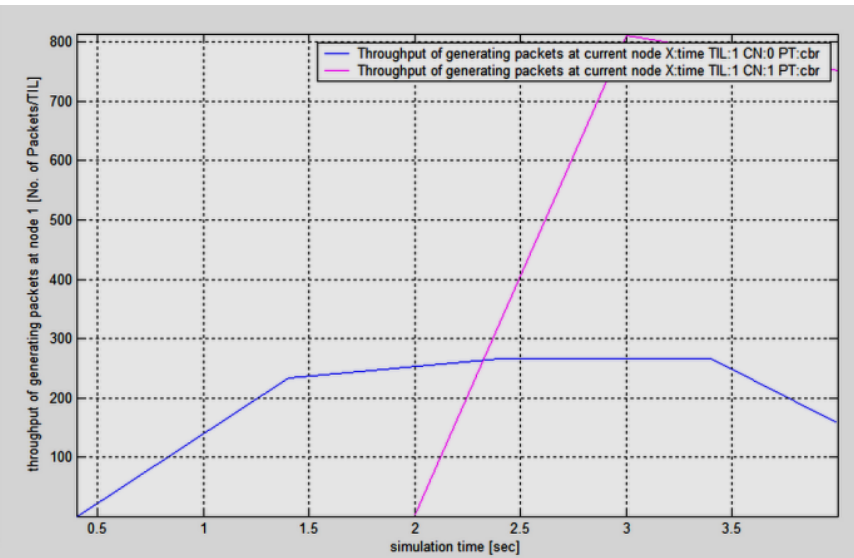

Fig.4 Throughput of generating packets at Source $1 \& 2$ Figure 4 shows number of packets generated at particular interval of time (i.e. throughput) for source1 (in blue) and source2 (in red). Maximum throughput of packet generation at source 1 and source 2 is 88 and 268 (approx.) respectively.

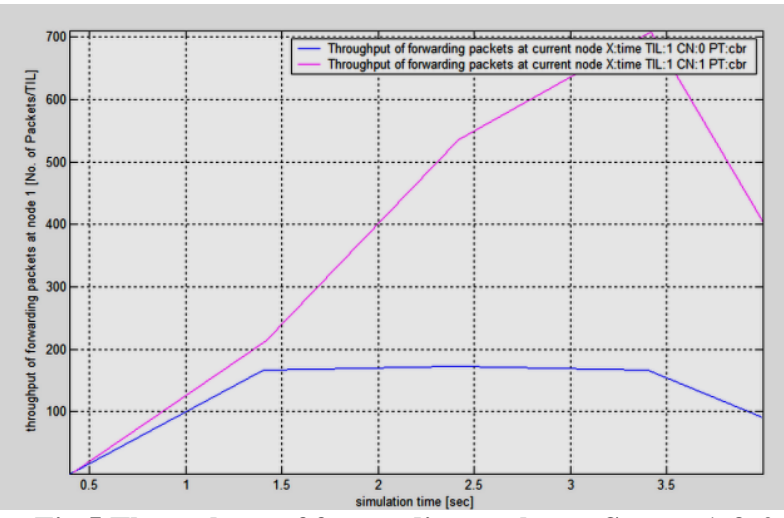

Fig.5 Throughput of forwarding packet at Source 1 \& 2

Figure 5 shows number of packets transferred at particular interval of time (i.e. throughput) for source1 (in blue) and source 2 (in red). Maximum throughput of forwarding packets at source 1 and source 2 is 53 and 207 (approx.) respectively which is relatively less than generated packets.

\subsection{Simulating PIM-DM Multicasting Network represented in $3 D$}

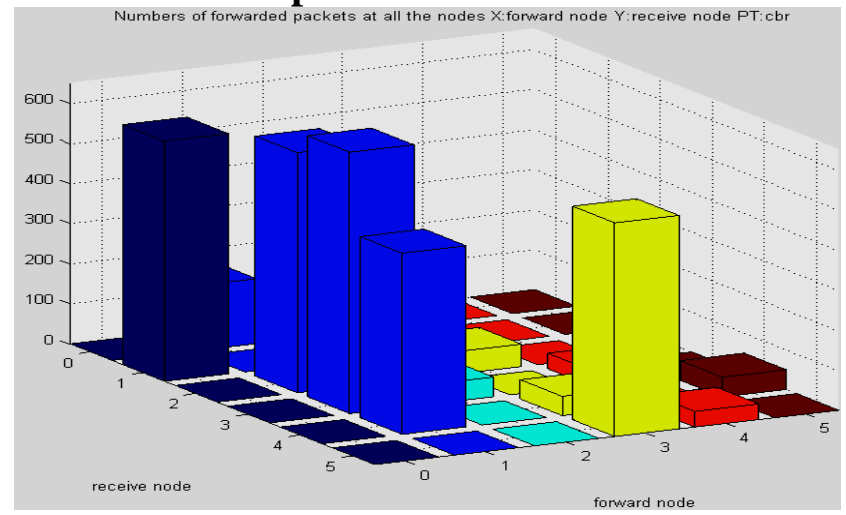

Fig.6 Number of forwarded packets at all Nodes
Figure 6 shows total packets forwarded while multicasting at each node. Total packets forwarded by source1 to source 2 are 545 (approx.) and total forwarded packets by source 2 to other nodes are 1300 (approx.). Node 5 receives packets through node3 and hence the graph shows a yellow color bar of forwarded packets by sender node3, receiver node5.

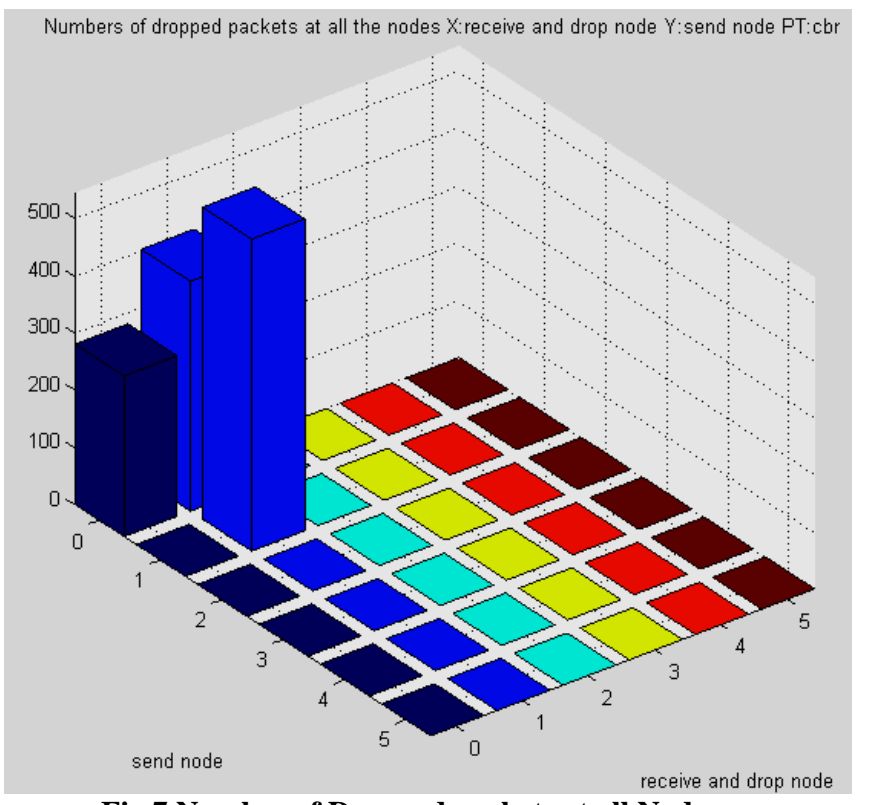

Fig.7 Number of Dropped packets at all Nodes

Figure 7 shows data packets drop out at source1 and source2. At source 1 total packets received and dropped by source 1 is 280 (approx.) and due to source 2 is 400 (approx.) (i.e. total data packets drop out is 680).

\subsection{Simulating BST Multicasting Network represented in 2D}

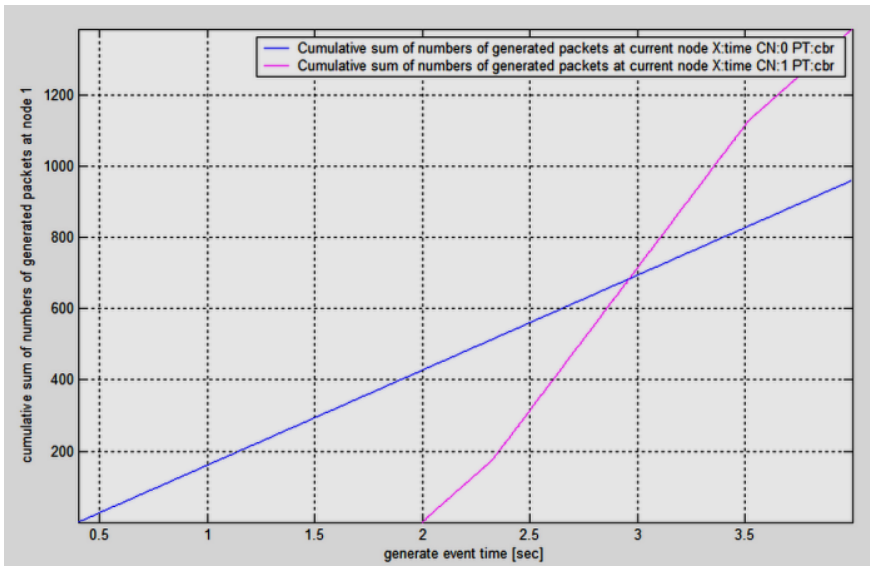

Fig.8 Packets Generated at Source 1 \& 2

Figure 8 shows the total CBR packets generated at source 1 and source2. Source1 is represented by blue and Source 2 by red. The figure represents that source2 start generating packet after 2seconds of source1 and generated upto 1300 data packets, while source1 upto 950 data packets (approx.). 


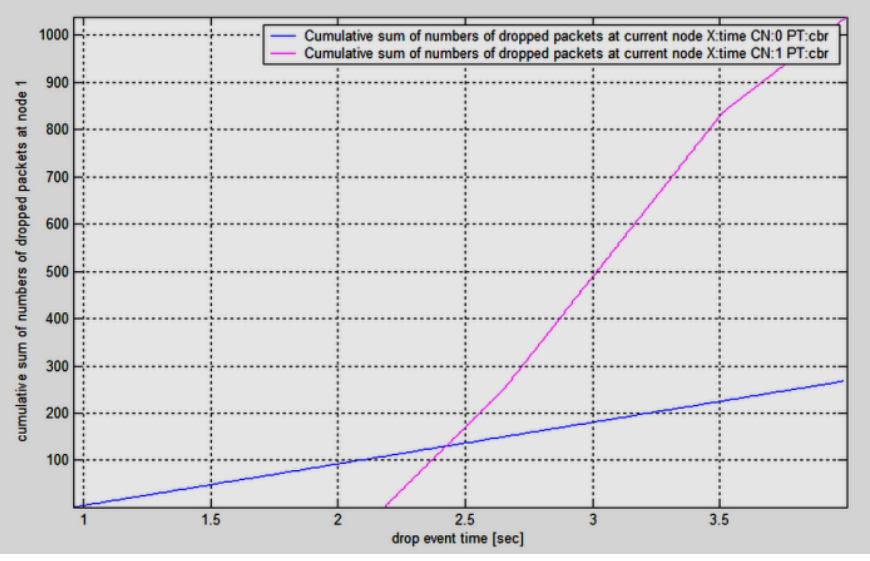

Fig.9 Packets dropped at Source $1 \& 2$

Figure 9 shows the drop out packets at source1 and source2 which is in the range of 280 and 1100 respectively. Dropping of packets starts at source 2 after $2.2 \mathrm{~s}$ of start of the simulation.

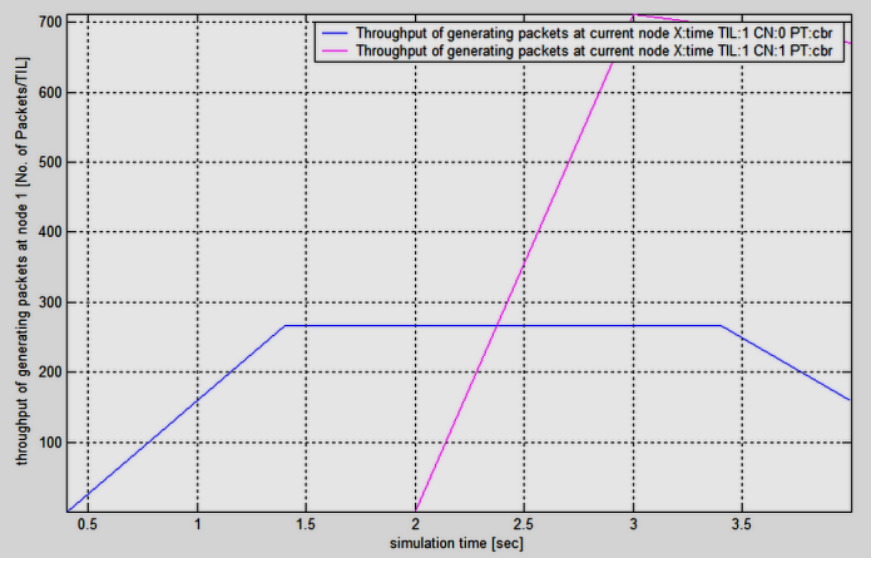

Fig.10 Throughput of generating packets at Source $1 \& 2$

Figure 10 shows number of packets generated at particular interval of time (i.e. throughput) for source1 (in blue) and source2 (in red). Maximum throughput of packet generation at source 1 and source 2 is 88 and 235 (approx.) respectively.

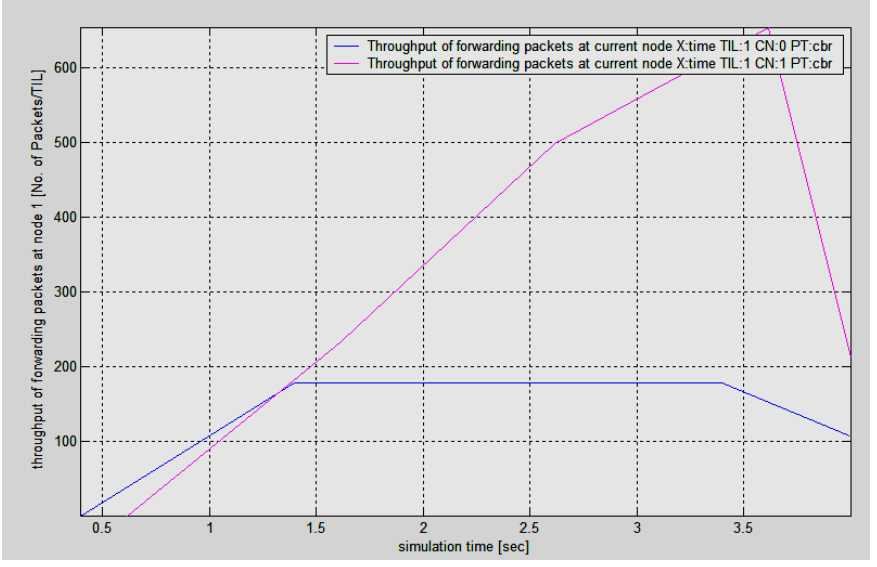

Fig.11 Throughput of forwarding packet at Source $1 \& 2$
Figure 11 shows number of packets transferred at particular interval of time (i.e. throughput) for source1 (in blue) and source2 (in red)

\subsection{Simulating BST Multicasting Network represented in 3D}

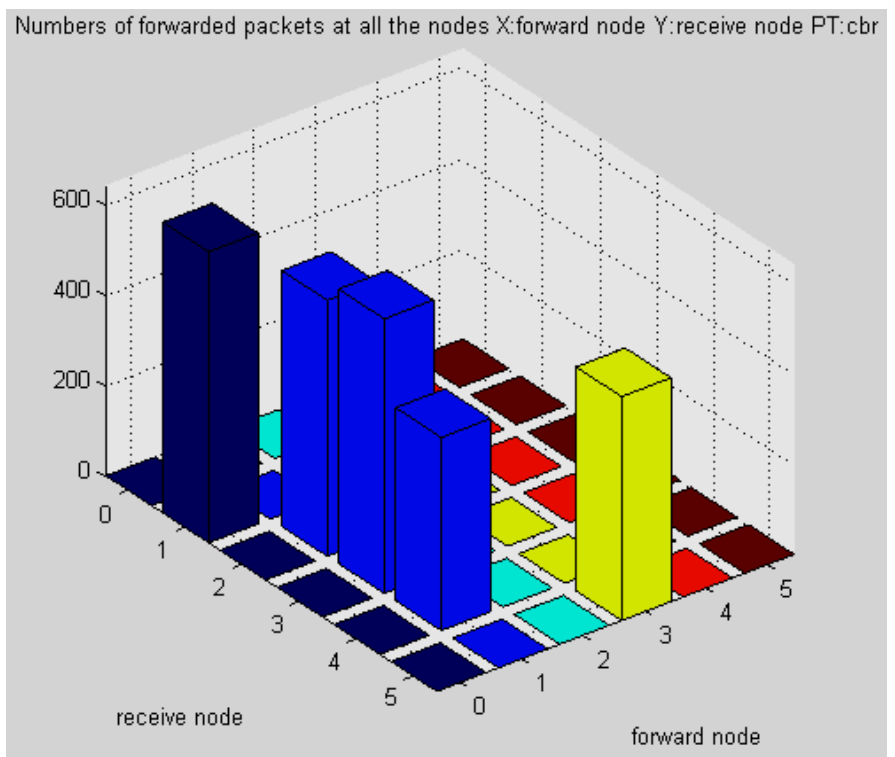

Fig.12 Number of forwarded packets at all Nodes

Figure 12 shows total packets forwarded while multicasting at each node. Total packets forwarded by source 1 to source 2 are 525 (approx.) and total forwarded packets by source 2 to other nodes are 850 (approx.). No data packet is forwarded to source1 by source 2 . Node 5 receives packets through node 3 and hence the graph shows a yellow color bar of forwarded packets by sender node3, receiver node5.

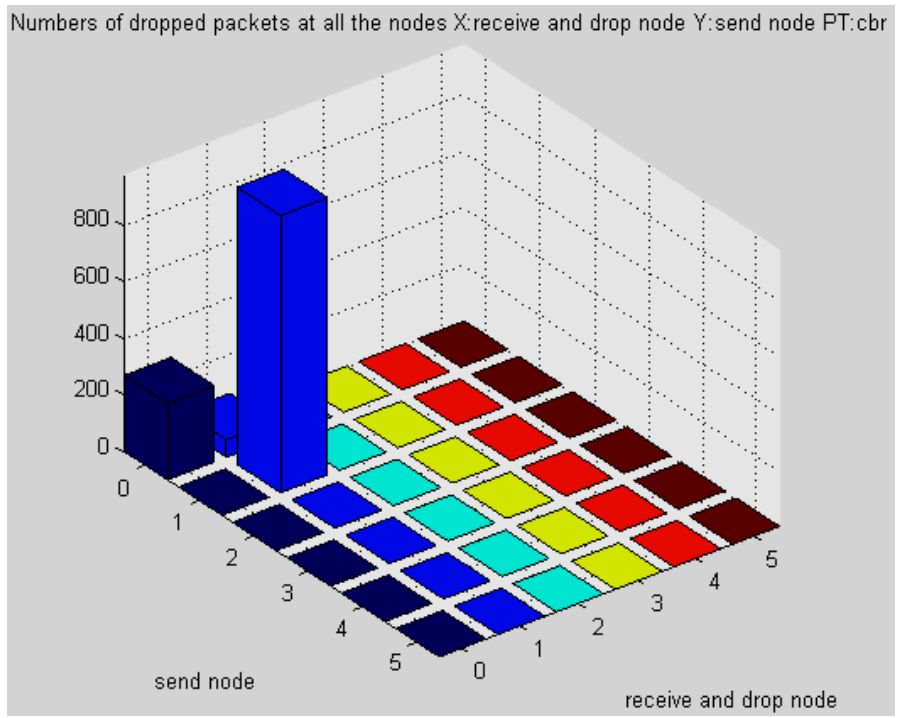

Fig.13 Number of Dropped packets at all Nodes 
Figure 13 shows data packets drop out at source1 and source2.

At source 1 total packets received and dropped by source 1 is 250 (approx) and due to source2 is 50 (approx) (i.e. total data packets drop out is 300). Hence decrease in percentage of drop of data packets $=((680-300) * 100) / 680=55.88235 \%$.

\section{SIMULATION RESULTS}

Table 1 Simulation information in PIM-DM

\begin{tabular}{|l|l|}
\hline Simulation length in seconds & 3.5994 \\
\hline Number of nodes & 6 \\
\hline Number of sending nodes & 6 \\
\hline Number of receiving nodes & 6 \\
\hline Number of generating packets & 3095 \\
\hline Number of sent packets & 2096 \\
\hline Number of forwarded packets & 1702 \\
\hline Number of dropped packets & 1303 \\
\hline Number of lost packets & 1575 \\
\hline Minimal packet size & 80 \\
\hline Maximal packet size & 210 \\
\hline Average packet size & 194.1892 \\
\hline Number of sent bytes & 372430 \\
\hline Number of forwarded bytes & 357420 \\
\hline Number of dropped bytes & 263490 \\
\hline Packets dropping nodes & 0 \& 1 \\
\hline
\end{tabular}

Table 1 shows Simulating information PIM-DM multicasting. The information displayed here includes total number of generated, sent, forwarded, dropped and lost packets.

Table 2 Simulation information in BST

\begin{tabular}{|l|l|}
\hline Simulation length in seconds & 3.5994 \\
\hline Number of nodes & 6 \\
\hline Number of sending nodes & 6 \\
\hline Number of receiving nodes & 2 \\
\hline Number of generating packets & 2352 \\
\hline Number of sent packets & 1033 \\
\hline Number of forwarded packets & 1712 \\
\hline Number of dropped packets & 1307 \\
\hline Number of lost packets & 1026 \\
\hline Minimal packet size & 80 \\
\hline Maximal packet size & 210 \\
\hline Average packet size & 209.7514 \\
\hline Number of sent bytes & 216020 \\
\hline Number of forwarded bytes & 359520 \\
\hline Number of dropped bytes & 274470 \\
\hline Packets dropping nodes & $0 \& 1$ \\
\hline
\end{tabular}

Table 2 shows Simulating information in BST multicasting. The information displayed here includes total number of generated, sent, forwarded, dropped and lost packets

\section{CONCLUSIONS}

This paper compares the multicasting protocol using NS2 simulation tool and analyze future aspects, As simulation results noted out to be data packets drop out of 680 in PIM-DM while 300 in BST. Hence $55.88235 \%$ decrease in drop out data packets for BST at source1. Also the throughput of multicasting BST network is mark ably high. In future we are looking towards changing the Rendezvous Point of network and expecting makeable difference.

\section{REFERENCES}

[1] K. Almeroth, "The evolution of multicast: From the MBone to interdomain multicast to Internet2 deployment," IEEE Network, vol. 14, pp. 10-20, January/February 2000.

[2] S S Nagamuthu Krishnan, Prof. (Dr).V Saravanan, "Defending Denial of Service: State Overload", Int. J. Advanced Networking and Applications Volume: 02, Issue: 03, Pages: 719-722 (2010).

[3] Mona A. Abou-Of, "Dynamic Bi-directional Multicast Shared Tree", 978-1-4244-5995-7/09@2009 IEEE.

[4] The ns Manual (formerly ns Notes and Documentation), The VINT Project A Collaboration between researchers at UC Berkeley, LBL, USC/ISI, and Xerox PARC. Kevin Fall hkfall@ee.lbl.govi, Editor Kannan Varadhan hkannan@catarina.usc.edui, Editor, May 9, 2010, page no 73

[5] Huang Shao-bin, Wu Yan-xia, Piao Xiu-feng, "Performance comparison of multicast routing protocols based on NS-2", Journal of Marine Science and Application, Vol. 4 No. 4, December 2005.

[6] RFE-3973, http://www.ietf.org/rfc/rfc3973.txt

[7] By Jim Guichard, Ivan Pepelnjak, Jeff Apcar, MPLS and VPN Architectures, Volume II, Cisco Press, June 06 2003, Page No 428.

[8] T. Bhaskar Reddy, Ali Ahammed, Reshma banu, "Performance Comparison of Active Queue Management Techniques", IJCSNC International Journal of Computer Science and Network Security, VOL.9 No.2, February 2009.

[9] S. mercy Shalinie, G. preetha, S. Dina Nidhya, Kiruthika Devi, "Fuzzy Adaptive Tuning of Router Buffers for Congestion Control", International Journal of Advancements in Technology,Vol 1, No 1 (June 2010) @IJoAT. 